\title{
Fiber Type Composition of Unoperated Rat Soleus and Extensor Digitorum Longus Muscles after Unilateral Isotransplantation of a Foreign Muscle in Long-Term Experiments
}

\author{
T. SOUKUP ${ }^{1}$, V. SMERDU ${ }^{2}$, G. ZACHAŘOVÁ ${ }^{1}$ \\ ${ }^{1}$ Institute of Physiology, Czech Academy of Sciences, Prague, Czech Republic, ${ }^{2}$ Institute of \\ Anatomy, Medical Faculty, Ljubljana, Slovenia
}

Received November 1, 2007

Accepted December 17, 2007

On-line April 1, 2008

\begin{abstract}
Summary
We examined the effects of the unilateral heterochronous isotransplantation on the fiber type composition and myosin heavy chain $(\mathrm{MyHC})$ isoform content of unoperated slow soleus and fast extensor digitorum longus muscles of female inbred Lewis strain rats. Comparison was made between "control" unoperated muscles of experimental rats (after intramuscular transplantation surgery) with the corresponding muscles of completely naive (unoperated) rats of three age groups (5-, 8and 14-month-old). This was done in order to ascertain whether these muscles can be used as reliable controls to the transplanted and host muscles for our ongoing grafting experiments. The fiber type composition was determined by assessing the histochemical reaction for myofibrillar adenosine triphosphatase, the MyHC isoform content was determined immunocytochemically using monoclonal antibodies specific to different $\mathrm{MyHC}$ isoforms and by sodium dodecyl sulphate polyacrylamide gel electrophoresis. Our experiments show that the heterochronous intramuscular isotransplantation procedure had no significant effect on the fiber type composition and MyHC isoform content of the "control" unoperated muscles of the experimental rats when compared to the corresponding muscles of the naive animals. Furthermore, the duration and type of isotransplantation did not also lead to differences among corresponding "control" muscles of experimental animals. We conclude that the unoperated muscles of the experimental rats can be used as controls in our current transplantation project dealing with long-term grafting experiments.
\end{abstract}

\section{Key words}

Muscle transplantations • Muscle fiber types • Effect of surgical treatment • mATPase and immunohistochemistry • Myosin heavy chains

\section{Corresponding author}

T. Soukup, Institute of Physiology, Academy of Sciences of the Czech Republic, Vídeňská 1083, CZ-142 20 Prague, Czech Republic. Fax: +420-241062488. E-mail: tsoukup@biomed.cas.cz

\section{Introduction}

Within the European Muscle Network project (MYORES, Network of Excellence no. 511978, Multiorganismic Approach to Study Normal and Aberrant Muscle Development, Function and Repair) we are studying the influence of innervation and thyroid hormones on the diversification of muscle fiber phenotype and MyHC isoform gene expression in regenerated muscles. For this purpose, a method of heterochronous isotransplantation was successfully introduced in rats (Jirmanová and Soukup 1995). In this experimental model two types of muscle transplantation are performed; the soleus (SOL) or extensor digitorum longus (EDL) muscles of young inbred Lewis rats are intramuscularly grafted either into the host EDL or SOL muscles of adult animals of the same strain. If the isotransplantation does not affect the unoperated SOL and EDL muscles of experimental rats in the long-term experiments, these muscles could be used as controls in the evaluation of the expected fiber type transitions in transplanted and host muscles.

Though the experimental animals usually recovered soon after the transplantation and their mobility seemed to be unaffected, the possibility that the operated animals did not load both limbs to the same extent should 
not be ignored. The operated limb, with the host muscle additionally "loaded" with the muscle graft, might be less intensively used and loaded than the contralateral unoperated one, which might thus have been overloaded. This could affect the MyHC isoform content and the fiber type composition of the control muscles, as has repeatedly been demonstrated that loading and unloading induces fiber type transitions, especially in slow muscles (Asmussen and Soukup 1991, Caiozzo et al. 1998, Goldspink et al. 1999, for review see Hämäläinen and Pette 1995, Talmadge 2000, Baldwin and Haddad 2001).

In our previous short-term study, performed on a smaller sample of adult rats (mean age of seven months), we demonstrated that the unilateral isotransplantation into the EDL muscle had no significant effect on the fiber type composition and $\mathrm{MyHC}$ isoform content of the unoperated muscles when compared to the homologous muscles of naive (unoperated) rats (Zachařová et al. 2005). However, altering experimental procedures, as well as possible long-term effects of muscle surgery on the "control" muscles were still possible. Therefore, in the present study, the fiber type composition and MyHC content of the unoperated muscles of the experimental animals were compared with that of the homonymous muscles of naive rats within the total range of 4 to 17 months. For the present study, we used a number of muscle samples, which enabled a statistical comparison of animals divided into three age groups. An additional aim of this study was to ascertain whether altering 1) the type of grafted and/or host muscle (SOL or EDL), 2) the location of the host muscle (right or left, or both), 3) the position of the control muscle according to the side of operation (ipsilateral or contralateral) or 4) the duration of the isotransplantation, has any effect on the control muscles in experimental rats.

Our data clearly show that the unilateral heterochronous isotransplantation procedure had no significant effect on the mean fiber type composition or mean MyHC isoform content of the "control" unoperated muscles of experimental rats when compared to the corresponding muscles of naive animals. The duration and the type of isotransplantation did not also lead to differences among the experimental muscles. Hence, the unoperated control muscles of the experimental animals can be used as controls in our current long-term grafting experiments in rats with the altered thyroid status (Zachařová et al. 1999, Soukup et al. 2001, Vadászová et al. 2004, 2006a,b Vadászová-Soukup and Soukup 2007, for review see Soukup and Jirmanová 2000).

\section{Methods}

\section{Muscle samples}

In this study SOL and EDL muscles of female inbred 4- to 17-month-old rats of the Lewis strain were analyzed. The animals were obtained from the authorized laboratory of the rat-breeding unit of the Institute of Physiology (Accreditation number 1020/491/A/00). The maintenance and handling of the experimental animals followed the EU Council Directive (86/609EEC) and the investigation was approved by the Expert Committee of the Institute of Physiology of the Academy of Sciences, Prague, Czech Republic. Inbred rats are used to suppress the graft rejection and to facilitate the animals' survival. The muscles were collected after intraperitoneal injections of Nembutal (sodium pentobarbital, $40 \mathrm{mg} / \mathrm{kg}$ ) and immediately frozen in liquid nitrogen, euthanasia was performed by an overdose of the anesthetic.

The SOL and EDL muscles were excised from both hind limbs of 38 naive and 36 experimental rats. In experimental animals, heterochronous isotransplantation of SOL or EDL muscles, excised from 3- to 4-week-old rats, was performed into the right and/or left EDL or SOL muscle of adult 2- to 3-month-old rats (for details see Jirmanová and Soukup 1995, 2001, Soukup and Novotová 2000). Most of these transplants were unilateral, but a few were bilateral. Unoperated and experimental animals were divided into three age groups with mean age approximately 5-, 8- and 14 months (Tables 1 and 2). Serial transverse cryosections $(10 \mu \mathrm{m})$ were prepared from each muscle belly and were analyzed for myofibrillar adenosine triphosphatase (mATPase) histochemistry and for MyHC immunocytochemistry. The remaining portions of the muscle were processed for SDS-PAGE.

\section{Myofibrillar ATPase histochemistry and immuno- cytochemistry}

Muscle fiber types were determined according to the activity of mATPase (E.C.3.6.1.3) after alkaline $(\mathrm{pH}$ 10.3) and acid ( $\mathrm{pH} 4.5$ and 4.3) preincubations (Guth and Samaha, 1970, Dubowitz and Brooke 1973). To reveal the MyHC isoform expression in muscle fibers, the muscle sections were incubated with different monoclonal antibodies (mAbs) specific for rat $\mathrm{MyHC}$ isoforms BA-D5 (MyHC-1), SC-71 (MyHC-2a), F-35 (all MyHC except 2x/d) and BF-F3 (MyHC-2b) (Schiaffino et al. 1986). These antibodies (supernatants) 
Table 1. Fiber type composition of soleus muscles.

\begin{tabular}{|c|c|c|c|c|c|c|c|c|}
\hline \multirow[b]{2}{*}{ Age (months) } & \multicolumn{2}{|c|}{ Age group 1} & \multicolumn{2}{|c|}{ Age group 2} & \multicolumn{2}{|c|}{ Age group 3} & \multicolumn{2}{|c|}{$\begin{array}{c}\text { Total } \\
\text { (from all muscles) }\end{array}$} \\
\hline & 4.8 & 4.9 & 7. 4 & 7.3 & 14.1 & 14. 1 & & \\
\hline & \pm 0.9 & \pm 0.9 & \pm 0.8 & \pm 1.4 & \pm 2.3 & \pm 2.2 & & \\
\hline & Norm & Exp & Norm & $\operatorname{Exp}$ & Norm & $\operatorname{Exp}$ & Norm & Exp \\
\hline & $\mathrm{n}=14$ & $\mathrm{n}=12$ & $\mathrm{n}=15$ & $n=24$ & $\mathrm{n}=28$ & $\mathrm{n}=18$ & $\mathrm{n}=57$ & $\mathrm{n}=54$ \\
\hline ATP 4.3 & 98.4 & 98.5 & 97.3 & 98.5 & 97.8 & 99.1 & 97.8 & 98.6 \\
\hline type 1 fibers & \pm 2.6 & \pm 2.2 & \pm 3.0 & \pm 2.4 & \pm 2.7 & \pm 1.5 & \pm 2.7 & \pm 1.9 \\
\hline ATP 10.3 & 1.6 & 1.5 & 2.7 & 1.5 & 2.2 & 0.9 & 2.2 & 1.4 \\
\hline type $2 A$ fibers & \pm 2.8 & \pm 2.4 & \pm 3.9 & \pm 1.5 & \pm 2.4 & \pm 1.7 & \pm 2.8 & \pm 1.8 \\
\hline anti-slow & 97.8 & 99 & 96.8 & 98.3 & 97.7 & 99.0 & 97.4 & 98.8 \\
\hline MyHC 1 & \pm 2.6 & \pm 2.1 & \pm 2.8 & \pm 1.5 & \pm 2.9 & \pm 2.2 & \pm 2.1 & \pm 2.2 \\
\hline anti-fast & 2.2 & 1 & 3.2 & 1.7 & 2.3 & 1 & 2. 6 & 1.3 \\
\hline$M y H C 2 A$ & \pm 4.1 & \pm 2.2 & \pm 3.5 & \pm 3.6 & \pm 2.8 & \pm 2.7 & \pm 3.1 & \pm 2.7 \\
\hline$B A-D 5$ & 97.8 & 98.8 & 97.5 & 98.3 & 98.5 & 99.6 & 97.9 & 99.0 \\
\hline MyHC 1 & \pm 2.2 & \pm 2.0 & \pm 2.4 & \pm 1.7 & \pm 3.2 & \pm 0.3 & \pm 2.2 & \pm 1.6 \\
\hline$S C-71$ & 2.2 & 1.2 & 2.5 & 1.7 & 1.5 & 0.4 & 2.1 & 1.0 \\
\hline$M y H C 2 A$ & \pm 3.2 & \pm 2.5 & \pm 3.0 & \pm 2.5 & \pm 3.2 & \pm 0.7 & \pm 3.0 & \pm 2.0 \\
\hline
\end{tabular}

Data are means \pm S.D. Fiber type composition of the right and left soleus (SOL) muscles of naive (Norm) and experimental (Exp) 4- to 17 -month-old rats subdivided into three age groups. In the experimental rats. either the EDL or SOL muscle was isotransplanted into the host left or right EDL or SOL muscle. The data are expressed as percentages of slow type 1 fibers demonstrated with mATPase reaction after acid preincubation at $\mathrm{pH} 4.3$ or with anti-slow mAb and specific anti-MyHC 1 (BA-D5) mAb and of fast type $2 \mathrm{~A}$ fibers stained by mATPase reaction after alkaline preincubation at $\mathrm{pH} 10.3$ or with anti-fast mAb and specific anti MyHC-2a (SC-71) mAb. Note that there are no significant differences $(p<0.05)$ for any evaluated reaction between the SOL muscles of naive and experimental rats. $\mathrm{n}=$ number of muscles.

were produced in the local Slovenian laboratory from corresponding cell lines provided from Deutsche Sammlung von Mikroorganismen und Zellkulturen (DSMZ, Braunschweig, Germany). Additionally, two commercially available mAbs, anti Slow (MyHC-1) and anti Fast (MyHC-2), both provided by Biotrend or Medac/Novocastra were used. The commercial mAbs were diluted 1:200, supernatants were used from undiluted to diluted 1:1000 in PBS containing 5\% bovine or donkey serum albumin (the appropriate dilutions were determined after testing hybridoma culture supernatants by immunocytochemistry). Primary antibody binding was revealed by the standard indirect peroxidase-antiperoxidase (PAP) technique (Sternberger 1986) using the PAP products (Dakopatts, Copenhagen, Denmark), by the avidin-biotin method using Vectastain ABC (Vector Laboratories, CA, USA), or by using donkey secondary antibody conjugated with HRP (Jackson Immunoresearch Laboratories, USA). The control sections incubated without primary $\mathrm{mAb}$ were devoid of positive staining.
Sodium dodecyl sulphate polyacrylamide gel electrophoresis (SDS-PAGE)

MyHC isoforms were either extracted as described earlier (Zachařová et al. 2005) or prepared as follows: muscle specimens were minced on ice and washed with five volumes of $20 \mathrm{mM} \mathrm{NaCl}, 5 \mathrm{mM}$ sodium phosphate and $1 \mathrm{mM}$ EGTA (pH 6.5). Myosin was extracted with $100 \mathrm{mM}$ sodium phosphate, $5 \mathrm{mM}$ EGTA and $1 \mathrm{mM}$ dithiothreitol $(\mathrm{pH} 8.5)$ and the supernatant containing myosin was diluted with one volume of glycerol $(87 \%)$ and stored at $-20^{\circ} \mathrm{C}$ (d'Albis et al. 1979). MyHC isoforms were separated by SDS-PAGE (Talmadge and Roy 1993). The separating gel contained $8 \%$ acrylamide, $0.16 \%$ bis-acrylamide, $37.4 \%$ glycerol, $0.4 \%$ SDS, $0.2 \mathrm{M}$ Tris-base (pH 8.8), $0.1 \mathrm{M}$ glycine, $0.1 \%$ ammonium persulphate and $0.06 \%$ tetramethylethylenediamine (TEMED). The stacking gel consisted of $4.2 \%$ acrylamide, $0.08 \%$ bis-acrylamide, $37.4 \%$ glycerol, $0.4 \%$ SDS, $70 \mathrm{mM}$ Tris-base (pH 6.8), $4 \mathrm{mM}$ EDTA, $0.1 \%$ ammonium persulphate (APS) and $0.12 \%$ TEMED. The inside running buffer consisted of $150 \mathrm{mM}$ 
Table 2. Fiber type composition of extensor digitorum longus muscles.

\begin{tabular}{|c|c|c|c|c|c|c|c|c|}
\hline \multirow[b]{2}{*}{ Age (months) } & \multicolumn{2}{|c|}{ Age group 1} & \multicolumn{2}{|c|}{ Age group 2} & \multicolumn{2}{|c|}{ Age group 3} & \multicolumn{2}{|c|}{$\begin{array}{c}\text { Total } \\
\text { (from all muscles) }\end{array}$} \\
\hline & 4.8 & 4. 9 & 7.3 & 8.9 & 14. 1 & 14.4 & & \\
\hline & \pm 0.9 & \pm 0.8 & \pm 0.8 & \pm 0.7 & \pm 2.3 & \pm 2.0 & & \\
\hline & Norm & $\operatorname{Exp}$ & Norm & $\operatorname{Exp}$ & Norm & $\operatorname{Exp}$ & Norm & Exp \\
\hline & $\mathrm{n}=13$ & $\mathrm{n}=12$ & $\mathrm{n}=17$ & $\mathrm{n}=22$ & $\mathrm{n}=25$ & $\mathrm{n}=11$ & $\mathrm{n}=55$ & $\mathrm{n}=45$ \\
\hline ATPase 4.3 & 5.9 & 5.7 & 5.4 & 5.4 & 7.3 & 5.5 & 6.2 & 5.6 \\
\hline type 1 fibers & \pm 0.7 & \pm 1.2 & \pm 2.3 & \pm 3.4 & \pm 2.5 & \pm 1.6 & \pm 1.9 & \pm 1.7 \\
\hline ATPase 4.5 & 16.9 & 17.2 & 18.3 & 17 & 16.2 & 19.6 & 17.2 & 18.2 \\
\hline type $2 A$ fibers & \pm 3.7 & \pm 2.3 & \pm 3.8 & \pm 4.2 & \pm 2.5 & \pm 3.0 & \pm 3.4 & \pm 3.1 \\
\hline ATPase 4.5 & 77.2 & 77.1 & 76.3 & 77.6 & 76.5 & 74.9 & 76.5 & 76.2 \\
\hline type $2 B$ fibers & \pm 3.9 & \pm 2.7 & \pm 4.1 & \pm 6.8 & \pm 2.5 & \pm 3.1 & \pm 3.5 & \pm 4.0 \\
\hline anti-slow & 5.8 & 4.9 & 6.1 & 5 & 6.5 & 5.3 & 6.1 & 5.1 \\
\hline type $1 \mathrm{MyHC}$ & \pm 1.4 & \pm 1.0 & \pm 2.9 & \pm 2.8 & \pm 1.6 & \pm 0.7 & \pm 2.2 & \pm 1.4 \\
\hline anti-fast & 94.2 & 95.1 & 93.9 & 95 & 93.5 & 94.7 & 93.9 & 94.9 \\
\hline $2 a+2 x / d+2 b$ & \pm 1.4 & \pm 1.4 & \pm 1.5 & \pm 4.2 & \pm 1.6 & \pm 0.9 & \pm 2.9 & \pm 1.6 \\
\hline$B A-D 5$ & 5.4 & 5.8 & 6.1 & 4. 8 & 7.3 & 4. 8 & 6.2 & 5.2 \\
\hline MyHC 1 & \pm 0.6 & \pm 2.6 & \pm 3.2 & \pm 2.8 & \pm 2.5 & \pm 0.8 & \pm 2.1 & \pm 2.0 \\
\hline$S C-71$ & 22.7 & 22.7 & 19.6 & 21.9 & 22 & 23.4 & 21.4 & 22.6 \\
\hline$M y H C 2 A$ & \pm 5.3 & \pm 5.7 & \pm 3.9 & \pm 2.5 & \pm 2.9 & \pm 5.1 & \pm 3.9 & \pm 5.4 \\
\hline$B F-35$ & 29.4 & 28.4 & 34.3 & 33.1 & 30 & 34.2 & 31.4 & 32.2 \\
\hline$M y H C 2 x / d$ & \pm 6.1 & \pm 2.9 & \pm 4.7 & \pm 6.8 & \pm 6.4 & \pm 8.3 & \pm 5.9 & \pm 6.9 \\
\hline$B F-F 3$ & 42.5 & 43.1 & 40 & 40.2 & 40.8 & 37.6 & 41 & 40.2 \\
\hline$M y H C 2 b$ & \pm 6.5 & \pm 4.4 & \pm 8.8 & \pm 6.4 & \pm 3.4 & \pm 8.4 & \pm 6.1 & \pm 7.3 \\
\hline
\end{tabular}

Data are means \pm S.D. The mean content of fiber types of the right and left extensor digitorum longus (EDL) muscles of naive (Norm) and experimental (Exp) 4- to 17-month-old rats subdivided into three age groups. In the experimental rats, either the EDL or SOL muscle was isotransplanted into the host left or right EDL or SOL muscle. The data are expressed as percentages of type $1,2 \mathrm{~A}$ and $2 \mathrm{~B}$ fibers classified according to the mATPase reaction or as percentages of fiber types determined by anti-slow and anti-fast MyHC mAbs and with specific mAbs recognizing MyHC-1 (BA-D5), -2a (SC-71), -2x/d (BF-35 as a negative marker) and -2b (BF-F3) MyHC isoforms. Note that no statistically significant difference $(p<0.05)$ was found for any evaluated reaction between the EDL muscles from naive and experimental rats. $\mathrm{n}=$ number of muscles.

glycine, $100 \mathrm{mM}$ Tris and $0.1 \%$ SDS and outside buffer was twice diluted. Electrophoresis was carried out at constant voltage $(70 \mathrm{~V})$ for $30 \mathrm{~h}$ at $4{ }^{\circ} \mathrm{C}$. After MyHC isoform separation, the gels were silver-stained (Blum et al. 1987) and individual bands were densitometrically evaluated using Quantity One Program (of Bio-Rad Laboratories) or using the AIDA 3.28 computer program (Advanced Image Data Analyzer, Germany).

\section{Quantitative morphological analysis}

The numerical $(\mathrm{N})$ and area $(\mathrm{A})$ proportions (\%) of muscle fiber types, determined according to the mATPase reaction and the MyHC isoform content were assessed by a 2D stereological method using the principles of unbiased counting frame and point counting
(Zachařová and Kubínová 1995, Zachařová et al. 1997, 2005). The stereological measurements were performed by the C.A.S.T. Grid System (Olympus, Albertslund, Denmark). The data are expressed as means \pm S.D. and the significance $(p<0.05)$ was evaluated by a suitable statistical test (Mann-Whitney, Kolmogorov-Smirnov or T-test) depending on the distribution normality of experimental data analyzed by the Shapiro-Wilks test.

\section{Results}

Fiber type composition and MyHC isoform content

As was demonstrated by mATPase reaction, the normal SOL muscles of the naive rats and the control SOL muscles of experimental rats were composed of two 


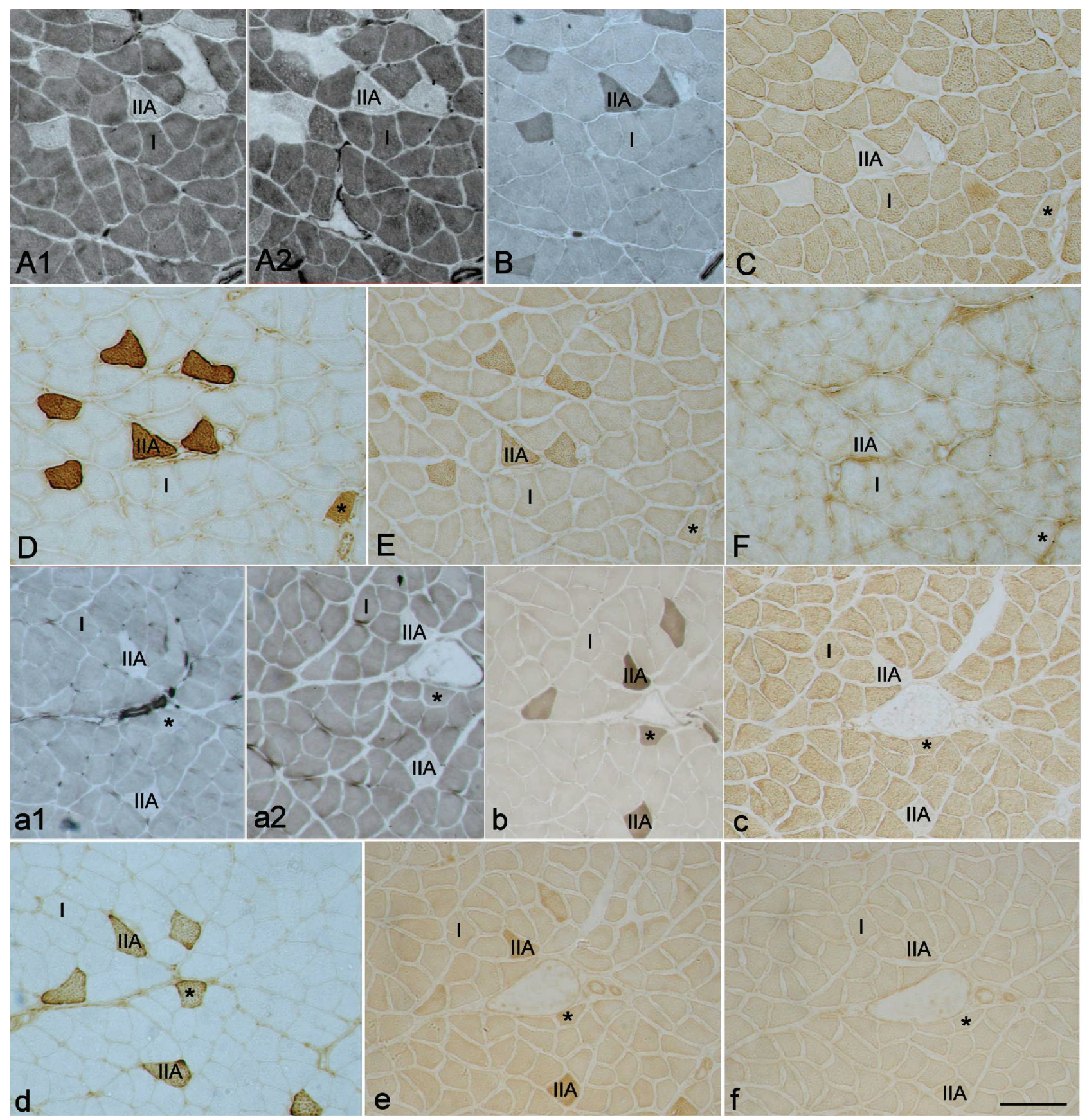

Fig. 1. Fiber types and $\mathrm{MyHC}$ isoforms in the SOL muscles of naive 8-month-old (A-F) and of 9.5-month-old experimental (a-f) female inbred Lewis strain rats. To show fiber types the sections were stained for mATPase after acid preincubation at pH 4.3 (A1, a1), 4.5 $(\mathrm{A} 2, \mathrm{a} 2)$ and after alkaline preincubation at $\mathrm{pH} 10.3(\mathrm{~B}, \mathrm{~b})$. MyHC isoforms were demonstrated with specific monoclonal antibodies recognizing slow type 1 (BA-D5, C, C), 2a (SC71, D, d), 1+2a+2b (negative marker for 2x/d, BF-35, E, e) and 2b (BF-F3, F, f) MyHC isoforms. Note that there are no differences in reactions of naive and experimental animals. Bar $=100 \mu \mathrm{m}$.

fiber types, type 1 and $2 \mathrm{~A}$; these were supplemented by scarce transitional hybrid type 2C (1C) fibers (Fig. 1). Type 1 fibers were stained positively after acid preincubations at $\mathrm{pH} 4.3$ and 4.5 for the mATPase reaction and with anti-slow and BA-D5 mAbs, while type 2A fibers were positively stained after the alkaline preincubation (they were negative after both 4.3 and 4.5 preincubations) and with anti-fast and SC-71 mAbs. All fibers of both types were stained by BF-35 mAb, specific to all MyHC isoforms except $-2 \mathrm{x} / \mathrm{d}$. Type $2 \mathrm{C}$ (1C) fibers were positively stained to a variable extent after acid and alkaline preincubations and, as expected, they co-expressed MyHC-1 and MyHC-2a isoforms. From the results of mATPase reaction and immunocytochemical reaction with BF-35 and BF-F3 mAbs, it is clear that no pure type $2 \mathrm{X} / \mathrm{D}$ and $2 \mathrm{~B}$ fibers were present in the SOL 


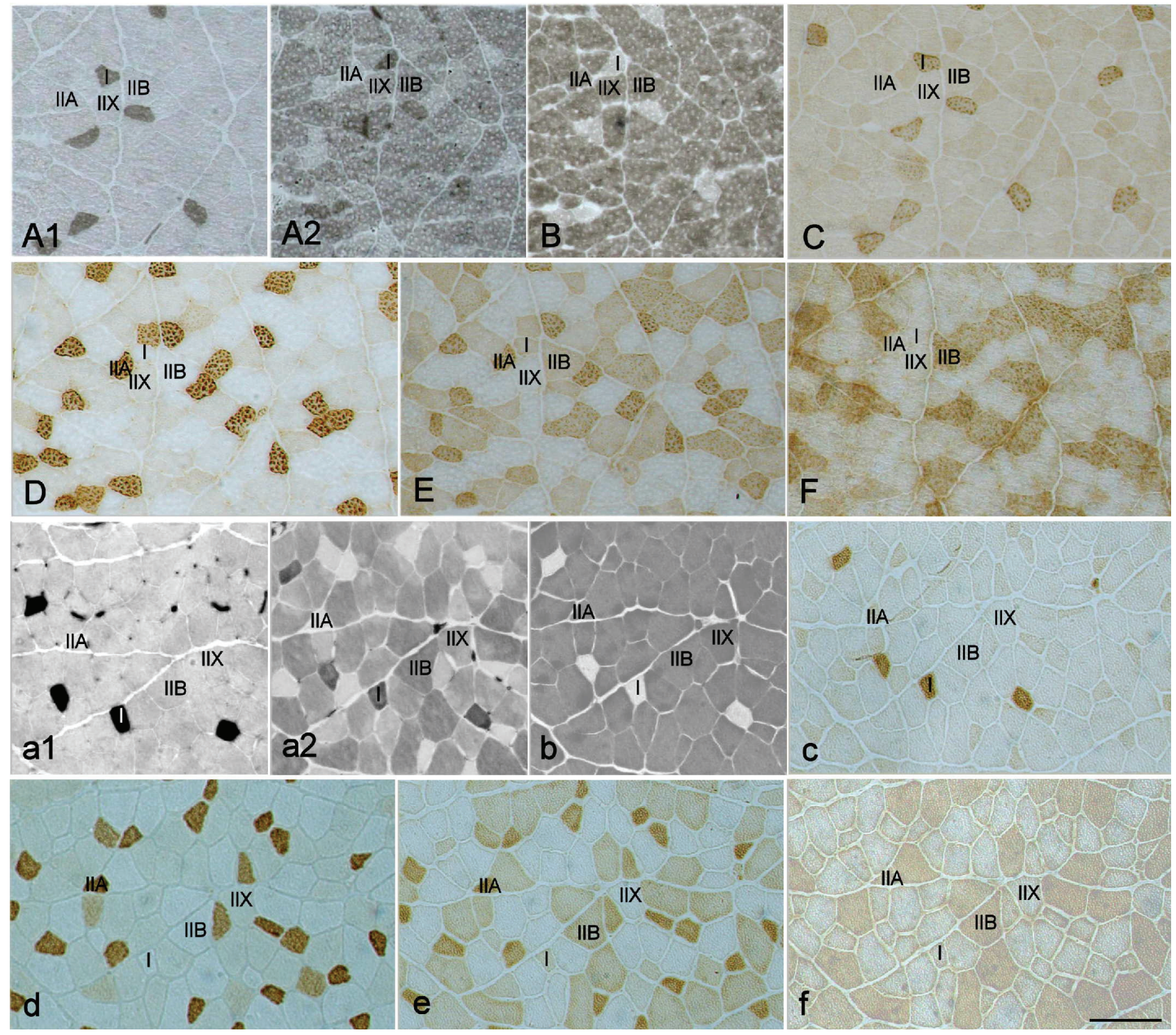

Fig. 2. Fiber types and MyHC isoforms in the EDL muscles of the naive 8-month-old (A-F) and 9.5-month-old experimental (a-f) female inbred Lewis strain rats. The fiber types were determined according to the mATPase reaction after acid pH 4.3 preincubation (A1, a1), $4.5(\mathrm{~A} 2, \mathrm{a} 2)$ and $10.3(\mathrm{~B}, \mathrm{~b})$ and MyHC isoforms were revealed with monoclonal antibodies specifically recognizing slow type 1 (BA-D5, $C, c), 2 a(S C-71, D, d), 1+2 a+2 b$ (negative marker for $2 x / d, B F-35, E, e)$ and $2 b(B F-F 3, F, f)$ MyHC isoforms. Note that there are no differences in reactions of naive and experimental animals. Bar $=100 \mu \mathrm{m}$.

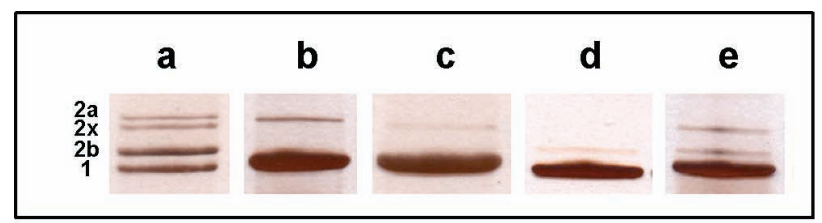

Fig. 3. MyHC isoforms in the SOL muscles of normal 4-month-old (b-c) and experimental 12-month-old (d-e) female inbred Lewis strain rats demonstrated by SDS-PAGE and silver stained. In lane a all four MyHC isoforms in the order top-to-bottom: MyHC- $2 a$, $-2 x / d,-2 b$ and -1 from a mixed sample of SOL and EDL muscle are shown as control to MyHC isoforms of SOL muscles (lanes be), containing different fast $\mathrm{MyHC}$ isoforms beside the predominating MyHC-1 isoform (the lowest band). In lane b SOL with commonly present MyHC-2a band is shown, in lanes c-e SOL muscles with "unusual" fast MyHC isoforms are presented: SOL with traces of MyHC-2x/d isoform (c), SOL with MyHC-2b isoform (d) and SOL with MyHC-2x/d and $-2 b$ isoforms (e). muscles of both naive and experimental rats (Fig. 1).

In the EDL muscles of naive and experimental rats, type $1,2 \mathrm{~A}$ and $2 \mathrm{~B}$ fibers were demonstrated by mATPase reaction (Fig. 2 A-B, a-b), while four fiber types (type 1,2A, 2X/D and 2B) were revealed by immunocytochemistry (Fig. 2 C-F, c-f). Type 1 and 2A fibers exhibited identical histochemical and immunocytochemical characteristics as in the SOL muscle and the histochemically defined $2 \mathrm{~B}$ fibers showed a fast phenotype characterized by high mATPase activity after preincubation at $\mathrm{pH} 10.3$ and moderate after preincubation at $\mathrm{pH}$ 4.5. Immunohistochemically, $\mathrm{MyHC}$ isoforms $2 \mathrm{x} / \mathrm{d}$ (the only negatively marked by BF-35 
$\mathrm{mAb}$ ) or $2 \mathrm{~b}$ (positively stained by BF-F3 mAb) were demonstrated in type $2 \mathrm{X} / \mathrm{D}$ and $2 \mathrm{~B}$ fibers, respectively. Similarly, four MyHC isoforms (MyHC1, 2a, 2x/d and 2b) were demonstrated by the SDS-PAGE technique (cf. mixed sample in Fig. 3a).

In the majority of the analyzed SOL muscles, SDS-PAGE revealed only MyHC-1 and $-2 \mathrm{a}$ isoforms (Fig. 3b). However, contrary to the histochemical and immunocytochemical techniques, SDS-PAGE revealed traces ( 1 to $6 \%$ ) of MyHC-2x/d and/or $-2 b$ isoforms in 10 (out of 57) normal SOL muscles from the naive rats and in seven (out of 54) unoperated control SOL muscles from the experimental rats (Fig. 3c-e).

Quantitative comparison between muscles of naive and experimental rats

The statistical analysis of SOL and EDL muscles (by the paired T-test and by the Mann-Whitney or Kolmogorov-Smirnov tests) revealed no significant difference in the mean fiber type composition and the mean MyHC isoform content between the right and left SOL and EDL muscles of naive and experimental rats. Therefore, for further comparison of studied parameters between the naive and experimental rats and among the respective age groups, the data of left and right SOL and EDL muscles were pooled.

Firstly, we found no significant difference in the analyzed parameters among the three age groups of naive or experimental rats (Tables 1 and 2).

Secondly, there was no significant difference in the analyzed parameters among any of the three age groups of experimental and naive rats (Tables 1 and 2).

Thirdly, therefore there was no significant difference in the analyzed parameters between corresponding muscles from experimental and naive animals when pooled within the whole age range of 4 to 17 months (Tables 1 and 2, Fig. 4).

Fourthly, the fact that the analyzed parameters of the three age groups of experimental rats were not statistically different shows that the duration of the postoperation period, lasting two to 15 months, had no effect on the fiber type composition and MyHC content in the control muscles of experimental animals.

The effect of different types of isotransplantation on the mean fiber type composition and the mean MyHC isoform content of control muscles in experimental animals

We found that the type of transplanted muscle (either SOL or EDL), as well as the type of the host muscle
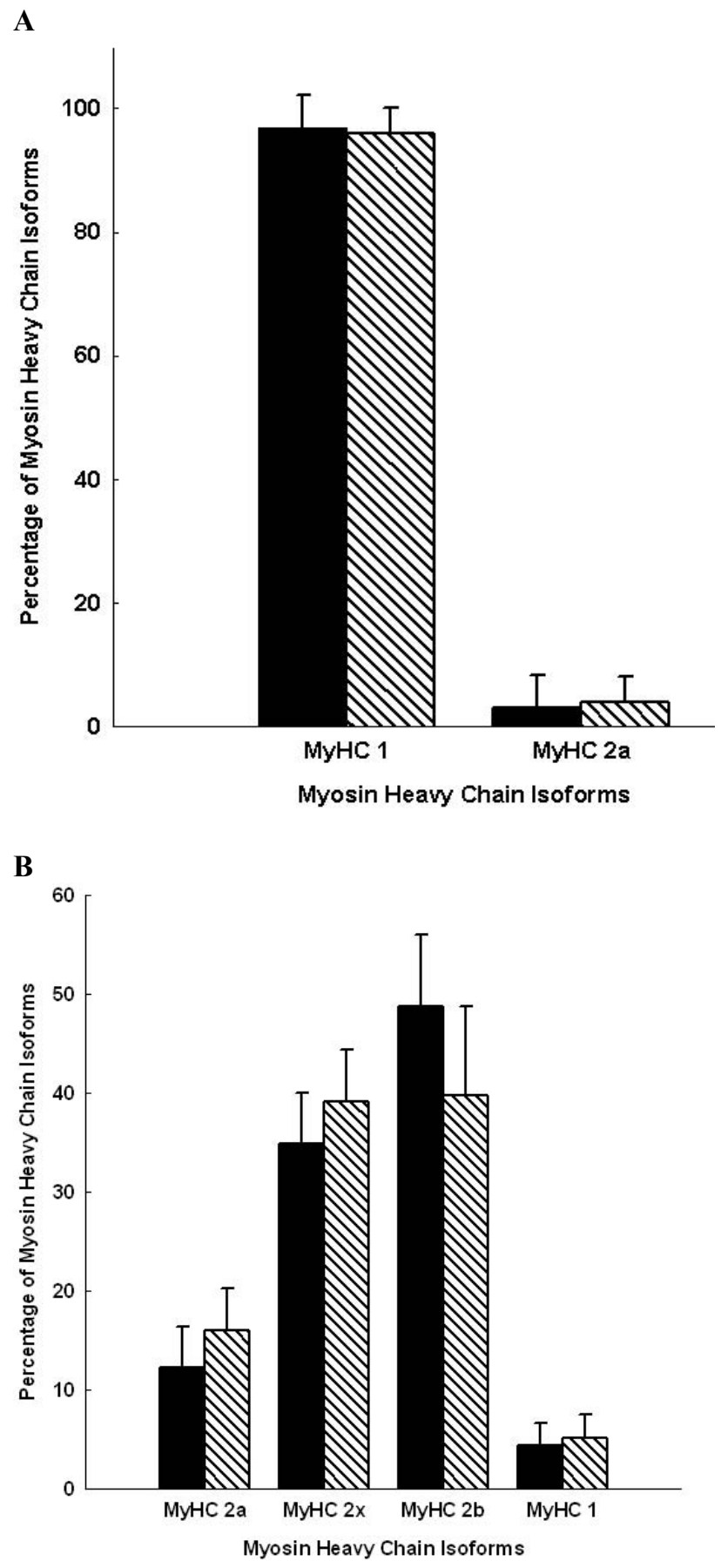

Fig. 4 A,B. The mean content of type 1 and $2 a$ MyHC isoforms as determined by SDS-PAGE in the rat SOL muscles $(\mathbf{A})$ and of type $1,2 \mathrm{a}, 2 \mathrm{x} / \mathrm{d}$ and $2 \mathrm{~b}$ isoforms in the EDL muscles (B) collected from hind limbs of naive (full columns) and experimental (after heterochronous isotransplantation, hatched columns) 4- to 17-month-old rats. Note that no statistically significant difference was found between naive and experimental rats. The number of analyzed muscles was 24 and 21 for SOL from naive and experimental rats, respectively and 32 and 28 for EDL from naive and experimental rats, respectively.

(either SOL or EDL) and the side of the transplantation (left or right) had no effect on the fiber type composition and MyHC isoform content of the control muscles. 
Furthermore, there were no significant differences in the fiber type and MyHC content of the control muscles between the contralateral and ipsilateral SOL or EDL muscles. Neither the duration of the isotransplantation nor the unilateral or bilateral transplantation affected the analyzed parameters of the unoperated control muscles.

\section{Discussion}

In the present study, we have demonstrated that fiber type composition and MyHC content of control (unoperated) SOL and EDL muscles excised from the experimental rats i) did not significantly differ from corresponding muscles excised from the naive rats, and ii) were not affected by the duration, the side of transplantation or the type of grafted and host muscle. Furthermore, no significant difference was found between analyzed parameters among any of the three age groups of the experimental and control animals. This clearly indicates that the composition of control muscles had not been affected by the duration and the type of isotransplantation (SOL/EDL, EDL/EDL, EDL/SOL or SOL/SOL), by the position in relation to the operated muscle (contralateral/ipsilateral) or if the operated leg was on the left or right side. Although we found no significant differences between the SOL and EDL muscles from naive and experimental rats on the average, there were, however, marked differences between left and right SOL muscles in some rats, as well as considerable differences among individual rats both in the naive and the experimental group. Nonetheless, our results demonstrate that the unoperated muscles of the experimental animals can be used, in general, as reliable controls for all types of regenerated muscle grafts and host muscles in our current analysis of thyroid hormone influence on the regulation of muscle phenotype (for review see Soukup and Jirmanová 2000).

In the long-lasting experimental study of muscle plasticity, various factors that may influence the fiber type composition and $\mathrm{MyHC}$ isoform content must be considered. In the present study, we therefore compared a large sample of muscles from experimental and naive rats in order to avoid a possible effect of the interindividual variability, differences between the right and the left side and the age, as well as of the duration and the type of isotransplantation in the experimental animals.

Firstly, the fiber type composition of muscles may change during the life of animals. The comparison among the three age groups of adult naive and experimental rats showed that the SOL and EDL fiber type composition does not change significantly after the fourth month of age. We have not included in this paper naive rats younger than four months, because the isotransplantation was performed in about 2-month-old rats and the graft regeneration was allowed at least for another two months in order to achieve its full regeneration. This means that the youngest experimental rats, available for comparison were 4 months old. The maximal time allowed for muscle regeneration in our grafting experiments was 15 months, thus the oldest experimental animals were about 17 months old. Nevertheless, the comparison of the oldest animals with other age groups did not reveal any significant differences, although the 17-month-old rats can already be regarded as "old" ones.

Secondly, though the analyzed animals were of the inbred strain (Lewis), it is possible that an individual variability in the fiber type composition and MyHC content of homonymous muscles among different rats can play a significant role. A possible variability between the right and the left muscles can also be expected in some rats. Although there were no significant differences between the mean values of the right and the left side, in five naive, as well as in five experimental rats, the difference in type 1 fibers and MyHC 1 content between their left and right SOL muscles was greater than $10 \%$. In contrast, in the EDL muscle, the difference in the total content of all fast isoforms between muscles from either leg were, in general, much smaller, even in rats with markedly different composition of the right and left SOL muscle. Our results also show that in the slow SOL muscle, in which MyHC 1 predominates, a certain degree of variability is possible even in the expression pattern of fast MyHC isoforms. As determined by the histochemical reaction for mATPase and by immunohistochemical reactions, the most commonly expressed fast isoform in SOL muscle is MyHC 2a. In addition, SDS-PAGE technique demonstrated the presence of minor amount of MyHC-2x/d and 2b isoforms in about $15 \%$ of the analyzed SOL muscles. Furthermore, the presence of MyHC 2x/d and 2b isoform mRNA transcripts in the SOL muscle was demonstrated by RT-PCR (Vadászová et al. 2006a) and recently also by real time RT-PCR (Žurmanová et al. 2008). However, the effect of fast MyHC isoforms on the SOL muscle inter-individual variability is apparently not significant, reflecting their relatively low proportion in this muscle. Our results demonstrated as well that SOL muscles containing 
limited amount of $2 \mathrm{x} / \mathrm{d}$ and/or $2 \mathrm{~b}$ MyHC isoform can be present in naive rats as frequently as in experimental animals. In addition to inter-individual variability, it is important to note that if different rat strains are used for comparative studies, the existing inter-strain differences also have to be considered (Soukup et al. 2002).

Thirdly, different experimental procedures may also affect the muscle fiber phenotype to a variable extent. However, our results do not indicate any influence of the duration of isotransplantation, as well as of variations of the isotransplantation method on the fiber type composition and $\mathrm{MyHC}$ isoform content in the "control" muscles of the experimental animals.

We therefore conclude that the unoperated SOL and EDL muscles of the experimental animals can be used as adequate controls to the host and regenerated muscle grafts in our ongoing transplantation studies.

\section{Conflict of Interest}

There is no conflict of interest.

\section{Acknowledgements}

We are grateful to Ms Martina Pytlová and Ms Kateřina Kopecká, to Mmes Andreja Vidmar, Majda ČrnakMaasarani, Ana Tomažinčič, Nataša Pollak and Daniela Horníková for their excellent technical assistance, $\mathrm{Mr}$ Marko Slak and Dr. Bohumil Štefl for computer analysis and Dr. Pavel Hník for critical reading of the manuscript and valuable comments. The study was supported by grants of the Grant Agency of the Czech Republic (304/05/0327, 304/08/0256), Czech-Slovenian Intergovernmental grant (02-2004-05), MSMT CR LC554, MYORES project contract number LSH-CT-2004-511978 and by the Research project AV0Z 50110509.

\section{References}

ASMUSSEN G, SOUKUP T: Arrest of developmental conversion of type II to type I fibers after suspension hypokinesia. Histochem J 23: 312-322, 1991.

BALDWIN K, HADDAD F: Plasticity in skeletal, cardiac, and smooth muscle. Invited review: Effects of different activity and inactivity paradigms on myosin heavy chain gene expression in striated muscles. J Appl Physiol 90: 345-357, 2001.

BLUM H, BEIER H, GROSS HJ: Improved silver staining of plant proteins, RNA and DNA in polyacrylamide gels. Electrophoresis 8: 93-99, 1987.

CAIOZZO VJ, BAKER MJ, BALDWIN KM: Novel transitions in MHC isoforms: separate and combined effects of thyroid hormone and mechanical unloading. J Appl Physiol 85: 2237-2248, 1998.

D'ALBIS A, PANTALONI C, BECHET JJ: An electrophoretic study of native myosin isoenzymes and of their subunit content. Eur J Biochem 99: 261-272, 1979.

DUBOWITZ V, BROOKE MH: Histological and histochemical stains and reactions. In: Muscle Biopsy: A Modern Approach. Major Problems in Neurology, Vol. 2, DUBOWITZ V, BROOKE MH (eds), WB Saunders, London, 1973, pp 20-33.

GOLDSPINK G: Changes in muscle mass and phenotype and the expression of autocrine and systemic growth factors by muscle in response to stretch and overload. J Anat 194: 323-334, 1999.

GUTH L, SAMAHA FJ: Procedure for the histochemical demonstration of actomyosin ATPase. Exp Neurol 28: 365367, 1970.

HÄMÄLÄINEN N, PETTE D: Patterns of myosin isoforms in mammalian skeletal muscle fibres. J Microsc Res Tech 30: 381-389, 1995.

JIRMANOVÁ I, SOUKUP T: Critical period in muscle spindle regeneration in grafts of developing rat muscle. Anat Embryol 192: 283-291, 1995.

JIRMANOVÁ I, SOUKUP T: Early changes in extrafusal and intrafusal muscle fibres following heterochronous isotransplantation. Acta Neuropathol 102: 473-484, 2001.

SCHIAFFINO S, SAGGIN L, VIEL A, AUSONI S, SARTORE S, GORZA L: Muscle fiber types identified by monoclonal antibodies to myosin heavy chains. In: Biochemical Aspects of Physical Exercise, BENZI G, PACKER L, SILIPRANDI N (eds), Elsevier, Amsterdam, 1986, pp 27-34.

SOUKUP T, JIRMANOVÁ I: Regulation of myosin expression in developing and regenerating extrafusal and intrafusal muscle fibres with special emphasis on the role of thyroid hormones. Physiol Res 49: 617-633, 2000. 
SOUKUP T, NOVOTOVÁ M: Ultrastructure and innervation of regenerated intrafusal muscle fibres in heterochronous isografts of the fast rat muscle. Acta Neuropathol 100: 435-444, 2000.

SOUKUP T, ZACHAŘOVÁ G, SMERDU V, JIRMANOVÁ I: Body, heart, thyroid gland and skeletal muscle weight changes in rats with altered thyroid status. Physiol Res 50: 619-626, 2001.

SOUKUP T, ZACHAŘOVÁ G, SMERDU V: Fibre type composition of soleus and extensor digitorum longus muscles in normal female inbred Lewis rats. Acta Histochem 104: 399-405, 2002.

STERNBERGER LA: Immunocytochemistry. Third edition, Wiley, New York, 1986.

TALMADGE RJ, ROY RR: Electrophoretic separation of the rat skeletal muscle myosin heavy-chain isoforms. $J$ Appl Physiol 75: 2337-2340, 1993.

TALMADGE RJ: Myosin heavy chain isoform expression following reduced neuromuscular activity: potential regulatory mechanisms. Muscle Nerve 23: 661-679, 2000.

VADÁSZOVÁ A, ZACHAŘOVÁ G, MACHÁČOVÁ K, JIRMANOVÁ I, SOUKUP T: Influence of thyroid status on the differentiation of slow and fast rat muscle phenotypes. Physiol Res 53 (Suppl 1): S57-S61, 2004.

VADÁSZOVÁ A, HUDECOVÁ S, KRIŽANOVÁ O, SOUKUP T: Levels of myosin heavy chain mRNA transcripts and content of protein isoforms in the slow soleus muscle of 7-month-old rats with altered thyroid status. Physiol Res 55: 221-225, 2006a.

VADÁSZOVÁ A, HUDECOVÁ S, KRIŽANOVÁ O, SOUKUP T: Levels of myosin heavy chain mRNA transcripts and protein isoforms in the fast extensor digitorum longus muscle of 7-month-old rats with chronic thyroid status alterations. Physiol Res 55: 707-710, 2006b.

VADÁSZOVÁ-SOUKUP A, SOUKUP T: Dual role of thyroid hormones in the rat soleus muscle MyHC isoform expression. Physiol Res 56: 833-836, 2007.

ZACHAŘOVÁ G, KUBÍNOVÁ L: Stereological methods based on point counting and unbiased counting frames for two-dimensional measurements in muscles: comparison with manual and image analysis methods. $J$ Muscle Res Cell Motil 16: 295-302, 1995.

ZACHAŘOVÁ G, KNOTKOVÁ-URBANCOVÁ J, HNÍK P, SOUKUP T: Nociceptive atrophy of the rat soleus muscle induced by bone fracture: a morphometric study. J Appl Physiol 82: 552-557, 1997.

ZACHAŘOVÁ G, MRÁČKOVÁ K, JIRMANOVÁ I, SOUKUP T: Stereological evaluation of the soleus muscle isografted into fast extensor digitorum longus (EDL) muscle in rats with different thyroid status. Gen Physiol Biophys 18 (Suppl 1): 84-86, 1999.

ZACHAŘOVÁ G, VADÁSZOVÁ A, SMERDU V, ASMUSSEN G, SOUKUP T: The effect of a unilateral muscle transplantation on the muscle fiber type and the MyHC isoform content in unoperated hind limb slow and fast muscles of the inbred Lewis rats. Physiol Res 54: 691-696, 2005.

ŽURMANOVÁ J, PƯTA F, STOPKOVÁ R, SOUKUP T: Real time RT-PCR with newly designed primers confirmed the presence of $2 \mathrm{~b}$ and $2 \mathrm{x} / \mathrm{d}$ myosin heavy chain mRNAs in the rat slow soleus muscle. Physiol Res 57: 973978, 2008. 